Supporting Information for

\title{
Ultrasensitive Magnetic Sensors Enabled by Heterogeneous \\ Integration of Graphene Hall Elements and Silicon Processing
}

\section{Circuits}

Tongyu Dai ${ }^{1 \dagger}$, Chengying Chen ${ }^{2 \dagger}$, Le Huang ${ }^{1 \dagger}$, Jianhua Jiang ${ }^{3}$, Lian-Mao Peng ${ }^{1 *}$, and Zhiyong Zhang ${ }^{*}$

${ }^{1}$ Key Laboratory for the Physics and Chemistry of Nanodevices and Center for Carbon-based Electronics, Department of Electronics, Peking University, Beijing 100871, China.

${ }^{2}$ School of Opto-electronic and Communication Engineering, Xiamen University of Technology, Fujian Xiamen, 361024, China

${ }^{3}$ Institute of Microelectronics, Chinese Academy of Sciences, Beijing 100029, China

*Correspondence to: E-mail: (Z. Z.) zyzhang@pku.edu.cn and (L.-M. P.)

lmpeng@pku.edu.cn

This file includes:

Fig. S1 to S4 
(a)

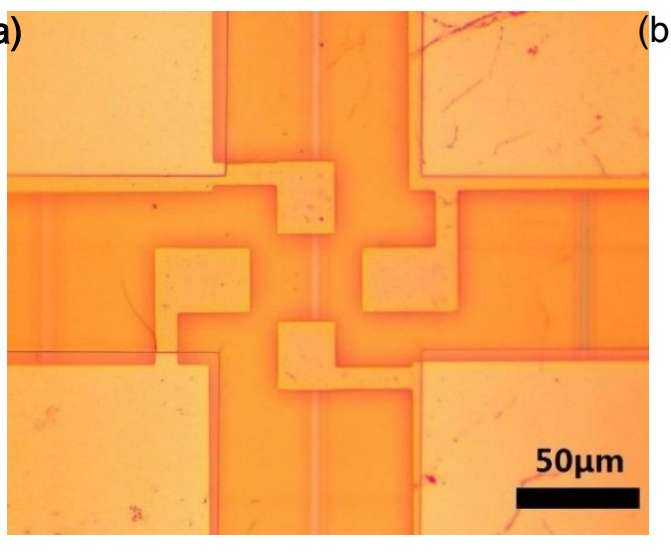

(b)

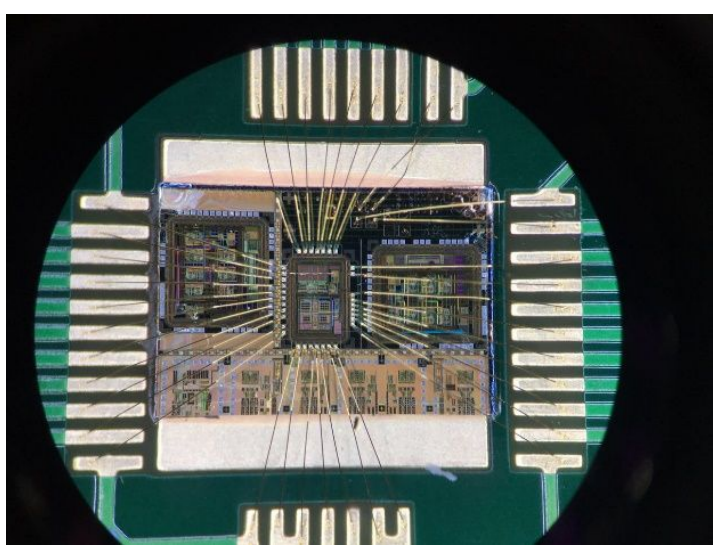

Figure S1 Optical photographs during preparation (a) Optical photographs of packaged devices. (b)The photo of graphene sensor after bonding.

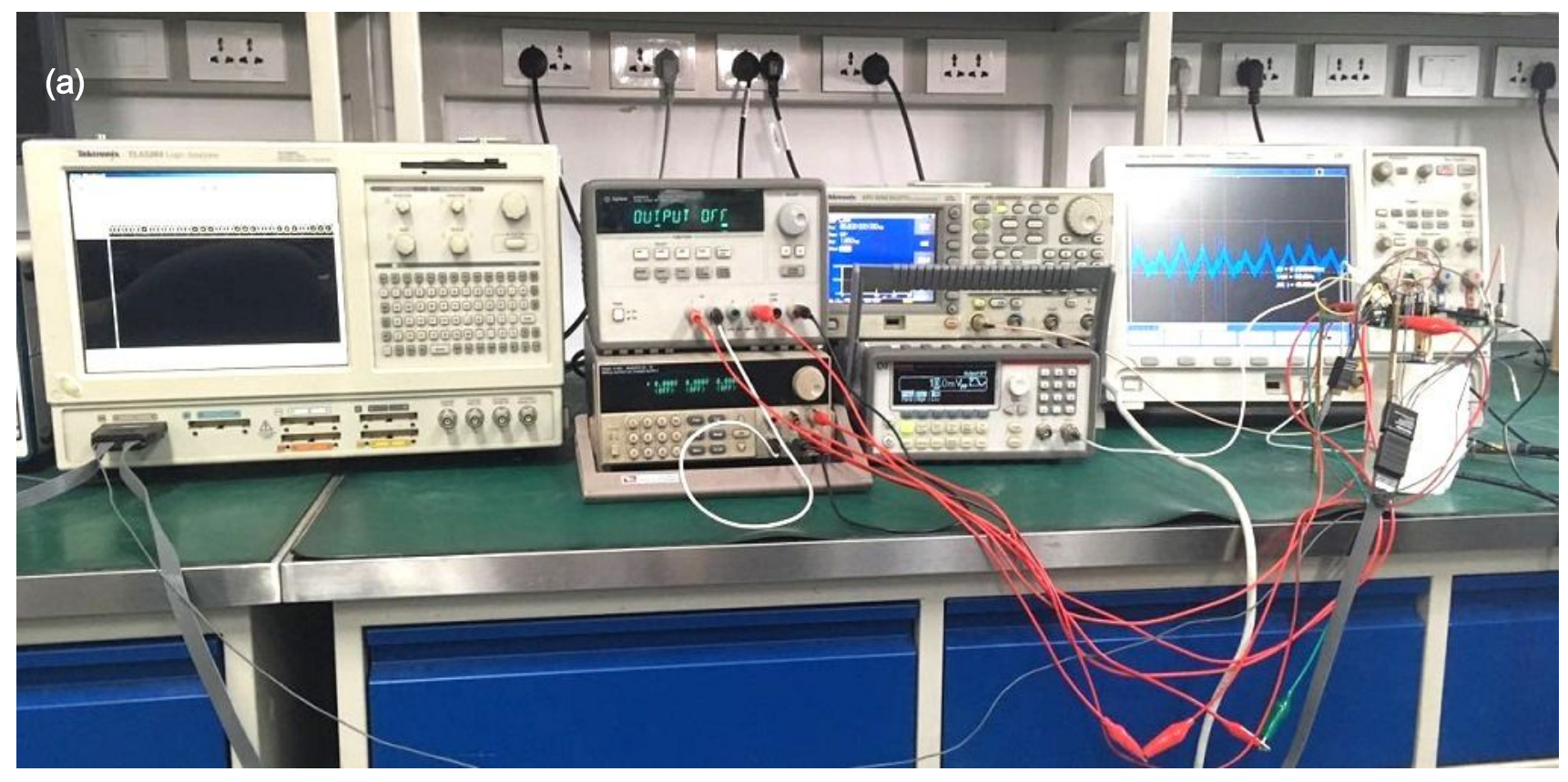


Figure S2 Measuring environment and instrument. The sensor is on the lower right. The white object beneath the sensor is a motor that drives the magnet to rotate. The figure displayed by the right oscilloscope is the current waveform.

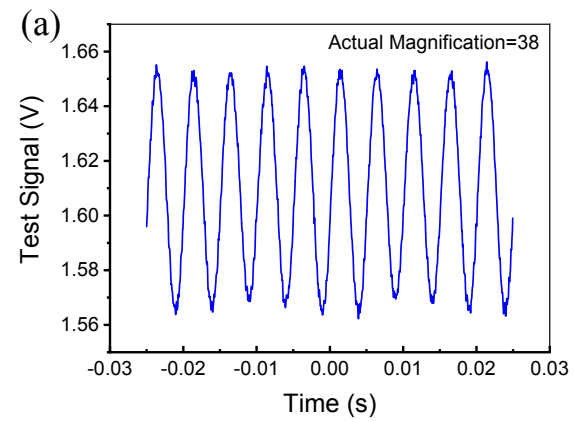

(b)
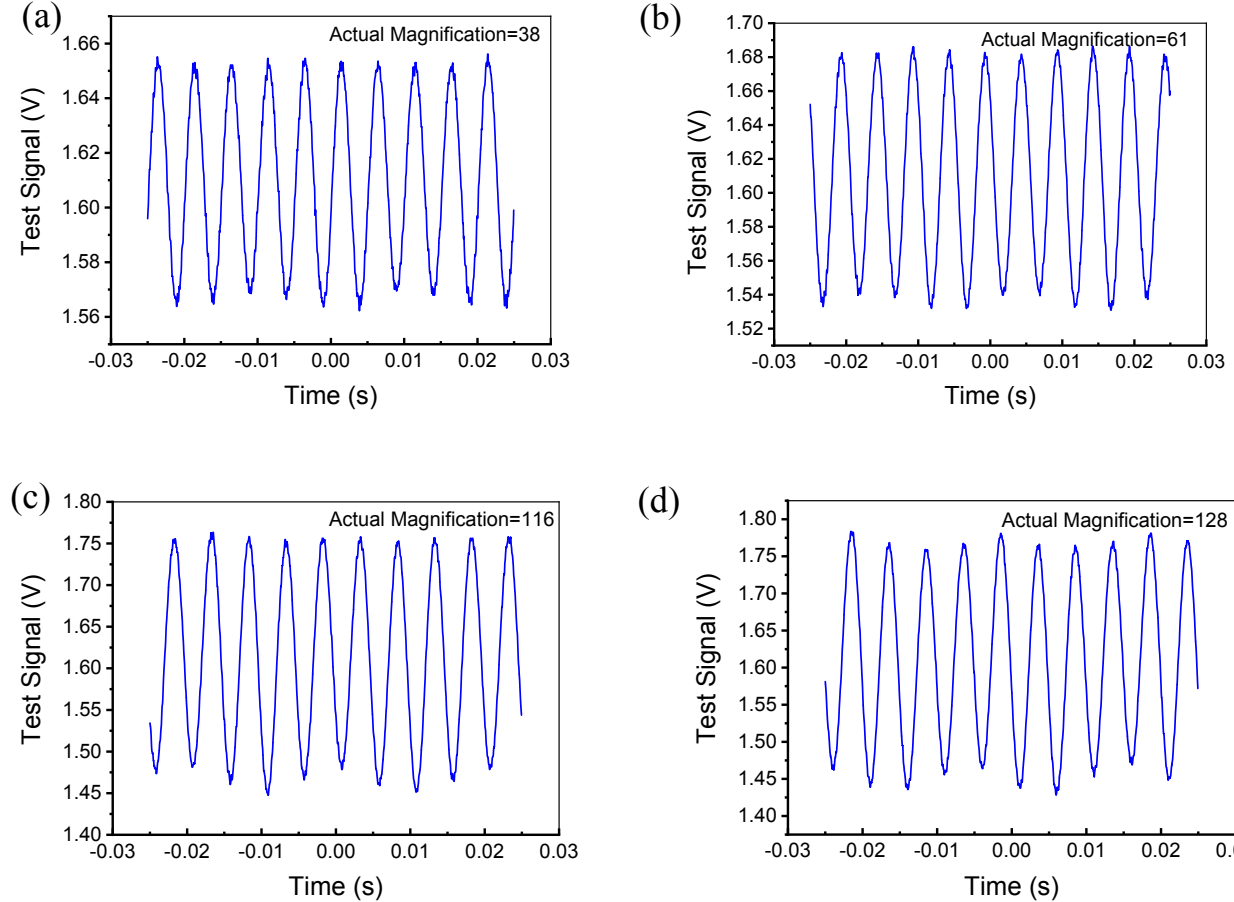

(d)

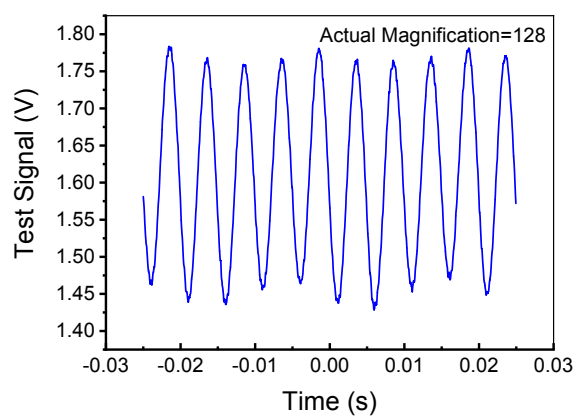

Figure S3 Testing the magnification of $200 \mathrm{~Hz}$ signal under four different feedback resistors
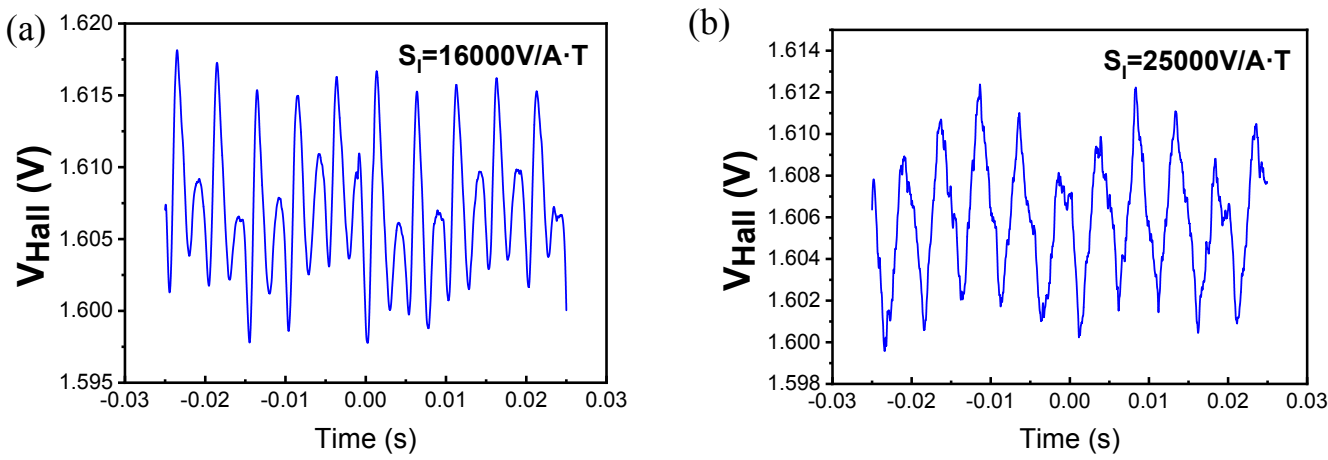

(c) 
(d)
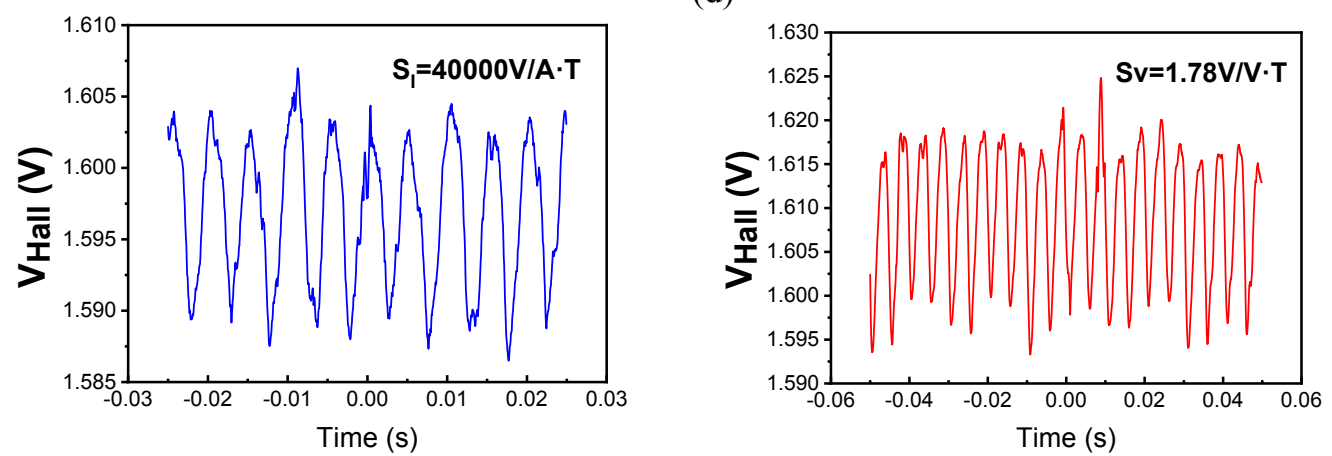

(e)

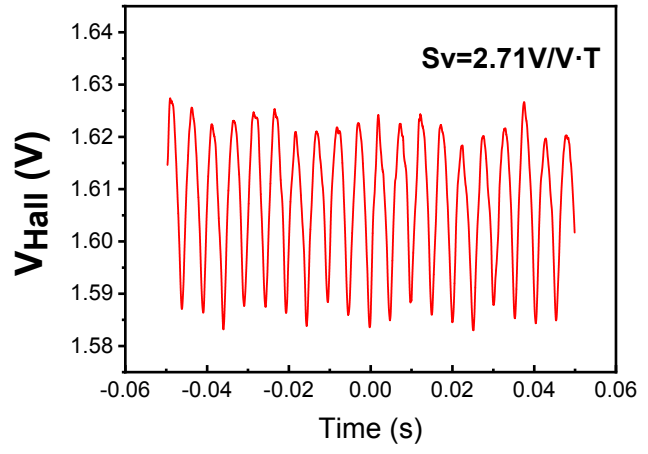

(f)

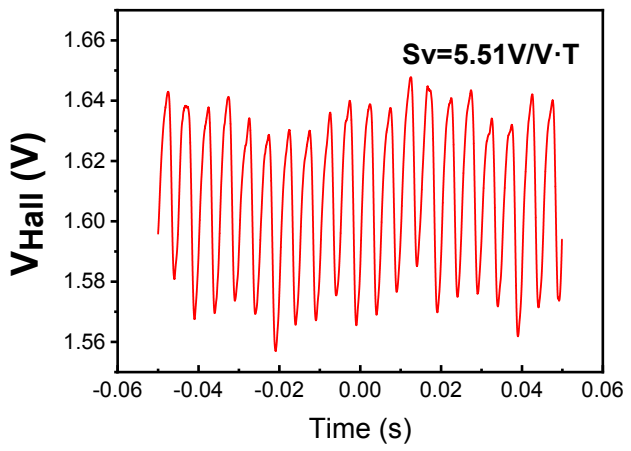

Figure S4 Testing at more amplification. (a)(b)(c) Three smaller magnification in fixed current mode (d)(e)(f) Three smaller magnification in fixed voltage mode. 


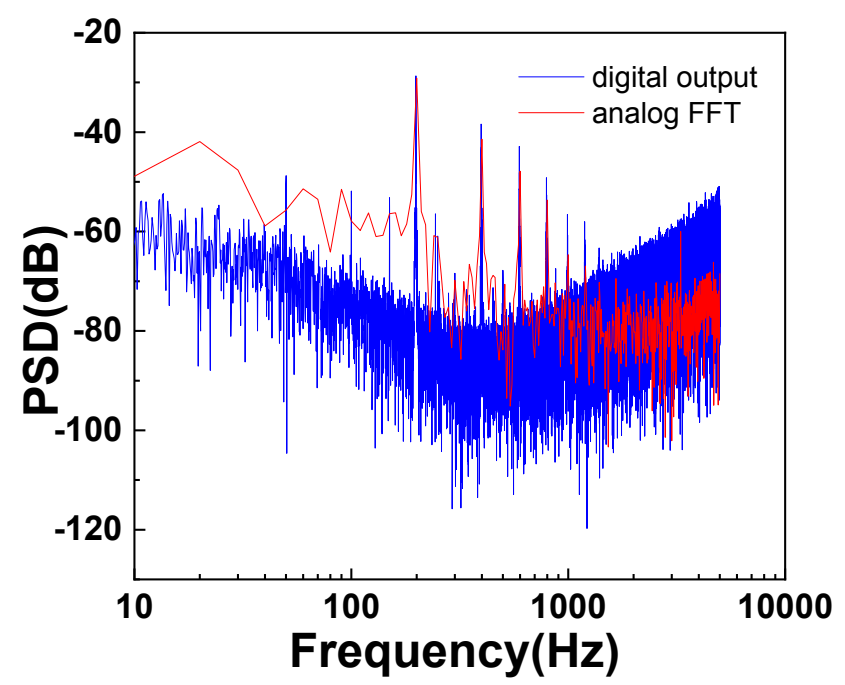

Figure S5 Spectrum comparison of analog signal and digital signal. The comparison proves that many sub-peaks of the final output signal are introduced before AD conversion, and the converted signal retains good signal frequency information in low frequency band. 